\title{
FATORES RELACIONADOS À OCORRÊNCIA DE QUEDAS EM CADEIRA DE RODAS: UMA REVISÃO
}

Paula Conceição Rocha de Oliveira (conceicao.rocha@unesp.br) - Universidade Estadual Paulista Júlio de Mesquita Filho (UNESP).

Fausto Orsi Medola (fausto.medola@unesp.br) - Universidade Estadual Paulista Júlio de Mesquita Filho (UNESP).

Marianna de Sousa Chaparro (marianna.chaparro@unesp.br) - Universidade Estadual Paulista Júlio de Mesquita Filho (UNESP).

\section{RESUMO}

Uma queda durante o uso de cadeira de rodas pode trazer danos físicos e psicológicos para seu usuário. A ocorrência pode se dar por diversos fatores e os desdobramentos para o indivíduo são recorrentemente estudados e discutidos. Com objetivo de gerar uma perspectiva para o design de cadeira de rodas, ambientes de uso e para entendimento do contexto de quedas, a presente revisão levanta uma discussão acerca das influências das experiências de queda relatadas em estudos. Foi realizada uma revisão de artigos científicos a partir de busca nas bases Scopus e PubMed. Como resultado, os materiais coletados elencaram aspectos do usuário, do ambiente e da cadeira de rodas que podem influenciar a probabilidade de uma queda ou que podem interferir nos sentimentos e lesões durante e após o acontecimento. Conclui-se que para melhorar o desempenho das cadeiras de rodas em relação a redução do risco de quedas, precisase entender a relação entre seus componentes, aspectos do usuário e do ambiente externo como informações inseparáveis e correlacionadas.

Palavras chave: cadeira de rodas; quedas; design de produto; ergonomia. 


\section{INTRODUÇÃO}

Em estudos sobre quedas em CR (cadeira de rodas), estas são definidas como eventos não planejados em que o indivíduo entrou em contato com superfície de suporte inferior (KHAN et al., 2019). Podendo segmentar eventos envolvidos, como em Chen et al. (2011), que coletou desequilíbrios, acidentes de colisão com objetos e operações perigosas com CR. Nesse contexto, em Chen et al. (2011) e Xiang, Chany e Smith et al. (2006) a maioria das quedas e desequilíbrios em CR resultaram em lesão. Em estudos preliminares acerca do assunto, Kirby et al. (1994) mostram que esses eventos são comuns, corroborado posteriormente por Berg et al. (2002), Forslund et al. (2017) e Khan et al. (2019). Eles apontam que lesões graves são incomuns, entretanto, qualquer acidente pode impactar a função social, saúde, segurança e aumentar o risco de futuros acidentes do indivíduo (CHEN et al., 2011).

As quedas em CR são reconhecidas como fatores significativos de complicações na saúde (KHAN et al., 2019). Na pesquisa nacional de Silveira (2019) a maioria dos participantes haviam sofrido um acidente na CR nos últimos 6 meses, diferindo entre ações causais como: subindo ou descendo um declive, transferência e propulsão, e fatores como: quebra na roda dianteira, pedras, buracos, quebra de componente da roda, desmaio e perda de equilíbrio. Existe a necessidade de coletar experiências por meios qualitativos - por exemplo, entrevistas semiestruturadas (NELSON et al. 2010), auto-relatos (BERG et al., 2002; NELSON et al. 2010) - em conjunto aos quantitativos, para compreender totalmente os resultados (SINGH et al., 2020), gerando também estudos mistos (RICE et al., 2019; SUNG et al., 2017).

A importância dessas abordagens se dá pelos diferentes preditores (FORSLUND et al., 2017), as quedas sendo eventos multifatoriais (BARBARESCHI; HOLLOWAY, 2020; RICE et al., 2019; SINGH et al., 2019) e complexos (XIANG; CHANY; SMITH, 2006). Para Singh et al. (2020) a estabilidade da CR, a satisfação do usuário e ergonomia são exemplos de fatores que influenciam a probabilidade de queda e lesões relacionadas. Soluções eficazes para melhorar as CR exigem esforço colaborativo de usuários, médicos, designers e outros profissionais (BARBARESCHI; HOLLOWAY, 2020). Entretanto, as tecnologias assistivas (TA) disponíveis são percebidas como ainda ineficazes no suporte durante transferências de CR, atividade mais desafiadora (BARBARESCHI; HOLLOWAY, 2020) e que frequentemente resulta em quedas (RICE et al., 2019; SINGH et al., 2020; SUNG et al., 2017; UMMAT; KIRBY, 1994). A capacidade de transferência independente é extremamente importante, afeta a vivência pessoal, social, e auto imagem do usuário (BARBARESCHI; HOLLOWAY, 2020). 
Experiências de quedas ainda são recorrentes no cotidiano de usuários de CR e podem gerar um risco desde lesões leves a graves. Essas vivências são complexas e multifatoriais, e essa característica deve ser considerada no design das CR e seus componentes de segurança, assim como em projetos de ambientes mais seguros. Por isso, deve-se gerar conhecimento acerca da relação entre as vivências de quedas, os indivíduos e suas CR, com intuito de melhorar o conhecimento de designers acerca dos fatores a serem considerados em um projeto de CR.

O objetivo desta revisão foi identificar as principais temáticas pesquisadas com relação à experiência de quedas com usuários de CR; e discutir os fatores do design do dispositivo, do usuário e do contexto em relação à ocorrência. Alguns aspectos dessas esferas podem influenciar a probabilidade de uma queda ou interferir nos sentimentos e lesões durante e após o acontecimento, sendo assuntos relevantes a serem considerados no design, prescrição e acompanhamento de CR.

\section{METODOLOGIA}

A coleta do material foi realizada em março de 2021, considerando a questão de pesquisa: "Quais os fatores envolvidos nas experiências de queda de usuários de cadeira de rodas?”. Os descritores utilizados nas bases Scopus e PubMed foram fall AND "wheelchair user", foram considerados os critérios de seleção: artigos de periódicos em inglês, artigos que tratassem de queda em CR, artigos que respondesse a questão de pesquisa. A filtragem inicial foi a leitura do título e do resumo, ao final: os artigos que se enquadraram foram lidos por completo e analisados no que tange a questão dessa pesquisa.

\section{RESULTADOS E DISCUSSÃO}

A busca retornou o total de 89 artigos, dos quais 69 foram excluídos por não corresponderem aos critérios de seleção, como resultado, foram lidos 20 textos completos, que resultaram em diversos aspectos para resposta da questão da pesquisa, convergindo para 3 principais esferas recorrentemente levantadas entre esses estudos e que geram interlocuções para a interação com a $\mathrm{CR}$, para o entendimento do indivíduo, com objetivo de dar apoio a projetos centrados no usuário e no bem-estar em aspectos globais da vida diária.

\subsection{Fatores do usuário}

A prevenção de quedas deve considerar os riscos de forma global, reconhecendo a situação atual do usuário e os diferentes fatores que interagem com ele, Singh et al. (2019) reconhece 
que o risco de queda é dinâmico e associado às mudanças em ambientes intrínsecos e extrínsecos, como fatores biológicos, comportamentais, sociais, econômicos, ambientais e da própria CR (SINGH et al., 2019). Fatores do usuário que parecem estar associados com aumento de risco de acidentes e lesões de quedas incluem: jovens, gênero masculino e paraplegia ou espinha bífida como razão de uso da CR, os fatores associados com a diminuição dos riscos incluem: esclerose múltipla, derrame, artrite como razão de uso da CR, propulsão por acompanhante e o uso de uma pessoa para ajudar em transferências (KIRBY et al., 1994). O estudo de Nelson et al. (2010) aponta fatores associados ao risco de quedas: histórico de quedas, dor, físico, uso de álcool, funcionalidade, habilidades com CR e acessibilidade em casa. Comprometimento do equilíbrio corporal, fraqueza muscular, espasticidade ou espasmos musculares, nível de função ou mobilidade e baixo desempenho em medidas clínicas são recorrentemente associados a quedas ou o risco (KHAN et al., 2019).

Sung et al. (2017) apresentam que indivíduos com esclerose múltipla tiveram maiores pontuações no Spinal Cord Injury-Fall Concerns Scale (SCI-FCS) - protocolo que avalia a preocupação com quedas em pessoas com lesão da medula espinhal (LME) que utilizam CR manuais (B0SSWELL-RUYS et al., 2010), e também pode ser utilizado com outras medidas, como em Rice et al. (2019), mostrando que a detecção de fatores de queda pode ser complexa e exigir diferentes abordagens. As causas mais frequentemente relatadas de quedas foram: mover-se rápido, inclinar-se além do centro de gravidade, erro de assistente, novo equipamento, baixo equilíbrio, distrações e pequenas colisões (SINGH et al., 2020) e para Kirby et al. (1994) e Ummat e Kirby (1994) foi verificado que lesões decorrentes de queda em CR se concentram na região da cabeça e pescoço.

Segundo Nelson et al. (2010) pessoas com LME usuárias de CR podem apresentar alto risco de quedas, independentemente da idade; para os autores, o número de anos de lesão é inversamente proporcional ao risco, podendo ser atribuído aos jovens e/ou pessoas ativas e atletas. A literatura também aponta a incidência de quedas relacionadas à prática esportiva em CR (SINGH et al., 2020). Na investigação de acidentes em atletas, Gaal et al. (1997) constatou que a maioria dos acidentes estavam relacionados a quedas, seguido de falha nos componentes. Considerando a prevalência das quedas nesse público, Forslund et al. (2017), em seu registro de quedas, os acidentes diretamente relacionados com atividades esportivas (32\% dos registros) foram excluídos da análise, com objetivo de avaliar apenas o ambiente cotidiano do usuário, sem interferência do cenário da prática esportiva. 
Em estudo de Sung et al. (2020), em amostra de usuários de CR manual e motorizada, a maioria relatou preocupação com a possibilidade de queda e que pararam atividades que costumavam fazer ou gostavam devido às preocupações em relação à queda. Os autores mostram que não há dados de diferença entre idade, condição de saúde, duração da deficiência, duração do uso de CR e número de quedas entre grupos com e sem medo de cair. Mas existem consequências psicossociais da queda, como medo, constrangimento e frustração, além de redução na participação de atividades diárias (KHAN et al., 2019). Rice et al. (2019) abordou a pior experiência de queda de usuários de CR, no estudo, a maioria dos participantes relataram o desenvolvimento de uma percepção da possibilidade de queda e medo da recorrência desse acontecimento após terem vivenciado suas piores quedas, gerando limitações na independência e mobilidade funcional. O medo pode estar associado à redução de atividade e influenciar a participação comunitária e qualidade de vida (SUNG et al., 2020). Foi encontrada uma diferença entre usuários com e sem medo de acordo com o tipo de CR -manual ou motorizada, em que usuários de motorizadas tem 6 vezes mais probabilidade de ter medo que os de manuais (SUNG et al.,2020). Uma possível explicação dos autores é que os indivíduos com CR motorizadas tendem a ter uma função física e mobilidade mais prejudicadas.

\subsection{Fatores do ambiente}

Para Forslund et al. (2017), existe diferença nos dados de queda entre estudos e fatores envolvidos devido a diferenças na metodologia de pesquisa, sendo a frequência e período de acompanhamento da amostra um dos fatores que diferem. Além desse cuidado, para Nelson et al. (2010) a avaliação de risco deve incluir condições ambientais como acessibilidade doméstica, embora nos primeiros estudos nesse sentido Kirby et al. (1994) verificaram que muitos dos acidentes aconteciam fora de casa, em rampas e no mesmo ano, Ummat e Kirby (1994) observaram que na maioria das quedas houve associação com causas externas, como por exemplo rampas.

Sung et al. (2017) avaliaram por meio de questão aberta as circunstâncias da queda mais recentes, que como resultado gerou três categorias principais apontadas pelos usuários de CR: ações/atividades contribuintes, ambiente do acontecimento, atribuições à queda (ex: condições da superfície). Como resultado, 5 ações ocorreram durante incidentes relatados: na maioria transferências em várias superfícies - cama, carro, sofá e chuveiro-, em menor número a propulsão da CR, locomoção por distâncias curtas, alcançar um objeto e por último ficando em pé (SUNG et al., 2017). 
Uma atividade recorrentemente associada às quedas na visão dos usuários é a transferência, caracterizada pela saída e entrada da cadeira para cama, carro, banheiro (FORSLUND et al., 2017; SINGH et al., 2020; UMMAT; KIRBY, 1994). No contexto da transferência entre artefatos e espaços, SINGH et al. (2020) aponta que a maioria dos seus participantes tiveram pelo menos 1 ocorrência de queda em 6 meses em razão de transferências ou terreno irregular. De acordo com Xiang, Chany e Smith (2006), a maioria das lesões dos usuários de CR infantis ocorreu fora de casa, em instituições/hospitais e ambientes com escadas, rampas e meios-fios, enquanto os ferimentos em adultos tinham maior ocorrência em casas, hospitais e instituições.

Em Forslund et al. (2017) a maioria das quedas ocorreu em ambientes fechados, mas em oposição, quedas relatadas em Rice et al. (2019) ocorreram com mais frequência na propulsão da CR em ambiente aberto, incluindo calçadas ou presença de detritos ou superfícies irregulares. Desse modo, não existe um consenso entre quais são os ambientes mais prováveis para acontecer uma queda, pois existe uma interferência da composição física e social desses ambientes, que podem se relacionar de maneiras particulares.

Como exposto, existe influência do ambiente físico, de apoio humano e atividades envolvidas em experiências de queda dos usuários de CR, mas os estudos ainda utilizam abordagens distintas para verificar essas influências. Alguns apontam o ambiente doméstico como mais propício a quedas, enquanto outros o ambiente externo. Acredita-se que além da divergência metodológica, outra razão dessa divergência é o ambiente estar ou não preparado para dar suporte e segurança física e social para usuários de CR.

\subsection{Fatores do design do dispositivo}

Fatores que parecem estar associados com aumento de riscos de queda incluem: a prescrição da CR, configurações (peso, cambagem, posição ou ajuste do eixo das rodas traseiras), uso diário, modo de propulsão, uso para recreação, formas de transferência, consertos inadequados (KIRBY et al., 1994). O estudo de Gaal et al. (1997) com atletas em CR indicou que a maioria das quedas estão relacionadas ao tipo de CR e de superfície. Kirby e Ackroyd-Stolarz (1995) verificaram que causas e proporção dos acidentes varia entre as diferentes TA de mobilidade (maior incidência nos scooters e CR motorizadas). Segundo eles, existem 4 classes de fatores de influência e agem de forma combinada: a engenharia, o ambiente, por último o usuário e o sistema (KIRBY; ACKROYD-SCOLARZ, 1995). A direção da queda também pode ser 
influenciada pela TA, a frontal é mais comum em CR manuais e motorizadas (KIRBY; ACKROYD-STOLARZ, 1995), mas para Gaal et al. (1997) a queda lateral se apresentou mais frequente entre CR motorizadas do que manuais (KIRBY; ACKROYD-SCOLARZ, 1995). Segundo Thomas, Borisoff e Sparrey et al. (2018) a probabilidade de queda para frente -com maior risco de lesões- foi significativamente reduzida pela menor velocidade, menores obstáculos, encosto reclinado e assento baixo.

Gaal et al. (1997) verificou que, em atletas, quedas por falhas de componentes aconteceram mais nas CR motorizadas, na maioria falhas de força ou sistema de controle, peças que as CR manuais não possuem, as quais apresentaram mais falhas no eixo traseiro e no pneu. Participantes do estudo de Singh et al. (2019) fizeram recomendações com base na própria experiência para reduzir riscos segundo a queda, tais como: plataforma giratória que forneça mais folga nas pernas durante transferência, rodas maiores com amortecimento, aros de propulsão mais largos para apoio extra ao se inclinar, maior inclinação no assento.

Fator observado acerca da engenharia é que rodas menores que $15 \mathrm{~cm}$ de diâmetro em CR manuais menores e rodízios sólidos geram mais quedas do que rodas maiores e/ou pneumáticas (GAAL et al., 1997). Menores rodízios e mais duros maximizam a manobrabilidade, mas podem ser parados mais facilmente em terrenos irregulares; por sua vez, a posição variável do eixo das rodas traseiras melhora a eficiência da impulsão, mas diminui a estabilidade posterior (GAAL et al., 1997). O menor comprimento -distância entre o rodízio dianteiro e o centro do eixo traseiro- da CR foi preditor de quedas para Nelson et al. (2010), por isso, é sugerida esta avaliação para análise de risco. O efeito da posição do eixo traseiro na estabilidade e manobrabilidade aponta a necessidade de desenvolvimento de eixos ajustáveis dinamicamente (THOMAS; BORISOFF; SPARREY, 2018). Gaal et al. (1997) afirma que mover o centro de gravidade para baixo e horizontalmente pode melhorar a habilidade ao lidar com obstáculos.

Para Thomas, Borisoff e Sparrey et al. (2018) as configurações relevantes para estabilidade da CR são: ângulo entre assento e encosto, posições do eixo traseiro, massa e posições do usuário, e esses aspectos sofrem influência principalmente de declives do solo e velocidade. Os autores apontam configurações e utilizações da CR que influenciam na direção da queda ou possibilidade de passar por obstáculos, salientam a importância do controle de velocidade e empinar (THOMAS; BORISOFF; SPARREY, 2018). 
Usuários de CR comentam que o peso e a largura de itens adicionais tornam a manobrabilidade extenuante (SINGH et al., 2019), embora seja importante adequar a CR com itens de segurança, na amostra de Chen et al. (2011) a maioria dos participantes não utilizava esses itens. No estudo de Singh et al. (2019) relacionou-se a manutenção dos freios e pressão dos pneus com a prevenção de problemas mecânicos e de distribuição de massa, corroborado por Chen et al. (2011), que aponta que indivíduos sem manutenção regular das CR e que não utilizam CR prescritas tinham maior risco de acidentes, por isso, Rice et al. (2019) afirmam que são necessárias travas mais robustas e manutenção adequada.

\section{CONSIDERAÇÕES FINAIS}

Com o exposto, foi elaborado um esquema (Fig. 1), resumindo as 3 esferas principais da experiência de queda: usuário, CR e ambiente, subdivididas em características específicas que se relacionam, influenciando em maior ou menor extensão o evento e a experiência durante e após. Acredita-se que irá auxiliar no entendimento a partir de uma perspectiva ampla, podendo contribuir para melhorias no design, prescrição, configuração e uso de CR. 
EXPERIÊNCIAS DE QUEDAS
Usuário
CR

\section{FATORES GERAIS}

Demográficos (deficiência, tempo de uso da CR, pratica de esportes)

Protocolos clínicos (funcionalidade, mobilidade, qualidade de vida)

Ambientes domésticos, de trabalho e lazer com acessibilidade

\section{Categoria da CR}

Configuração geral (eixo ajustável, tipo das rodas, ângulos e alturas de encosto e assento)

Configuração extra (componentes de segurança, apoios para transferências)

\section{DADOS ESPECIFICOS}

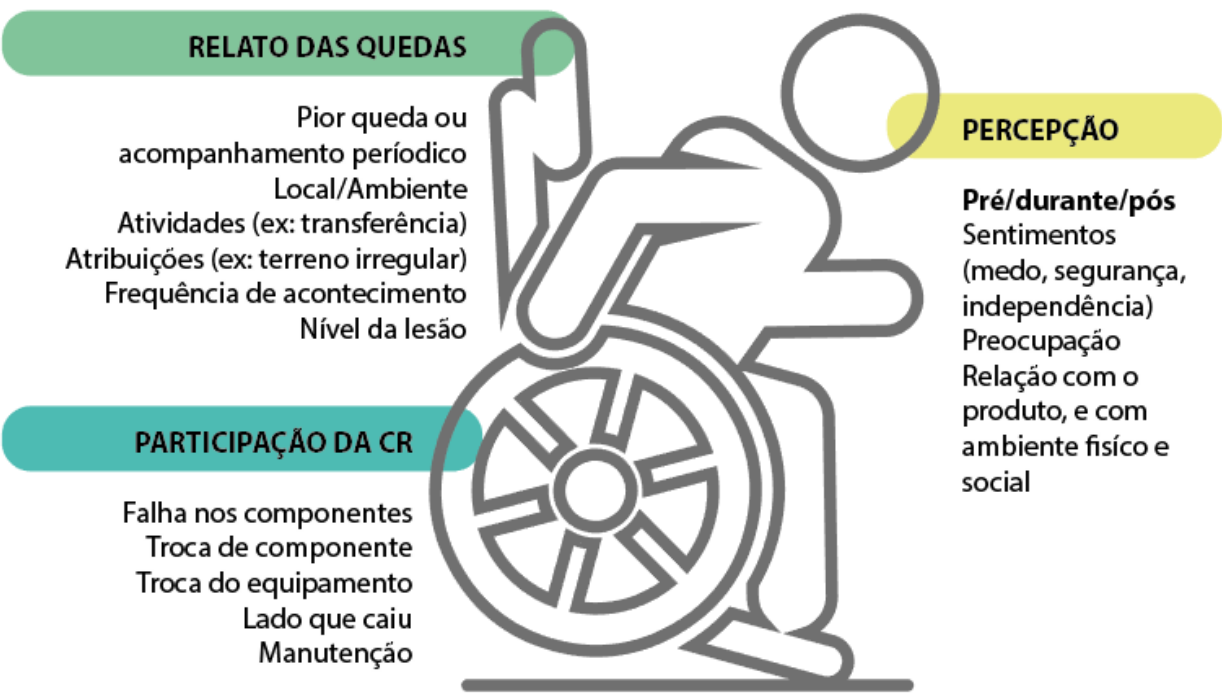

FIGURA 1 - Fatores envolvidos em experiências de queda. Fonte: Os autores

Fatores do usuário, ambiente físico, configuração da CR, uso e manutenção podem influenciar o risco de quedas, a gravidade das lesões, as características do evento e suas consequências. Nota-se uma dualidade entre ajustar a estabilidade e a manobrabilidade. $\mathrm{O}$ design deve considerar inseparavelmente o projeto estrutural da CR, as características do usuário e do ambiente cotidiano, para garantir a melhor configuração em relação à estabilidade, manobrabilidade, segurança e independência.

Indicam-se estudos futuros que associam percepção de queda e configuração da CR em aspectos mais específicos, como: adição ou remoção de itens e possibilidade de ajustes. Acredita-se que possa existir diferença em lidar com momentos de queda entre usuários com diferentes domínios com CR - como empinar e frear. Além disso, percebe-se lacunas em 
entender se esse acontecimento afeta a relação e a interação com o artefato durante atividades da vida diária.

\section{AGRADECIMENTOS}

O presente estudo teve apoio da Coordenação de Aperfeiçoamento de Pessoal de Nível Superior (CAPES - Processo 88887.597667/2021-00); e Conselho Nacional de Desenvolvimento Científico e Tecnológico (CNPq - Processo 310661 / 2017-0).

\section{REFERÊNCIAS}

BARBARESCHI, G.; HOLLOWAY, C. Understanding independent wheelchair transfers. Perspectives from stakeholders. Disability and Rehabilitation: assistive technology, v. 15, n. 5, p. 545-552. 2020. DOI: 10.1080/17483107.2019.1594407.

BERG, K.; HINES, M.; ALLEN, S. Wheelchair Users at Home: Few Home Modifications and Many Injurious Falls. American Journal of Public Health, v. 92, n. 1, p. 48. 2002. DOI: 10.2105/ajph.92.1.48.

BOSWELL-RUYS, C.; HARVEY, L.; DELBAERE, K.; LORD, S. A falls Concern Scale for people with spinal cord injury. Spinal Cord, v. 48, p. 704-709. 2010. DOI: 10.1038/sc.2010.1.

CHEN, W.; JANG, Y.; WANG, J.; HUANG, W.; CHANG, C.; MAO, H.; WANG, Y. Wheelchair-Related Accidents: Relationship With Wheelchair-Using Behavior in Active Community Wheelchair Users. Archives of Physical Medicine and Rehabilitation, v.92, n. 6, p. 892-898. 2011. DOI: 10.1016/j.apmr.2011.01.008.

FORSLUND, E. B.; JORGENSEN, V.; FRANZÉN, E.; OPHEIM, A.; SEIGER, A.; STAHLE, A.; HULTLING, C.; STANGHELLE, J. K.; ROALDSEN, K. S.; WAHMAN, K. High Incidence Of Falls And Fall-Related Injuries In Wheelchair Users With Spinal Cord Injury: A Prospective Study Of Risk Indicators. Journal of Rehabilitation Medicine, v. 49, n. 2, p. 144-151. 2017. DOI: 10.2340/16501977-2177.

GAAL, R. P.; REBHOLTZ, N.; HOTCHKISS, R. D.; PFAELZER, P. F. Wheelchair rider injuries: causes and consequences for wheelchair design and selection. Journal of Rehabilitation Research and Development, v. 34, n. 1, p. 58-71. 1997.

KIRBY, L.; ACKROYD-STOLARZ, S. A.; BROWN, M. G.; KIRKLAND, S. A.; MACLEOD, D. A. Wheelchairrelated accidents caused by tips and falls among noninstitutionalized users of manually propelled wheelchairs in nova scotia. American Journal of Physical Medicine \& Rehabilitation, v.73, n.5, p. 319-330. 1994. DOI: 10.1097/00002060-199409000-00004.

KIRBY, L.; ACKROYD-STOLARZ, S. A. Wheelchair safety - adverse reports to the United States food and drug administration. American Journal of Physical Medicine \& Rehabilitation, v.74, n.4, p.308-312. 1995. DOI: 10.1097/00002060-199507000-00009.

KHAN, A.; PUJOL, C.; LAYLOR, M.; UNIC, N.; PAKOSH, M.; DAWE, J.; MUSSELMAN, K. E. Falls after spinal cord injury: a systematic review and meta-analysis of incidence proportion and contributing factors. International. Springer Nature. Spinal Cord, v. 57, n. 7. p. 526-539. 2019. DOI: 10.1038/s41393-019-0274-4.

NELSON, A. L.; GROER, S.; PALACIOS, P.; MITCHELL, D.; SABHARWAL, S.; KIRBY, R. LEE; GAVINDRESCHNACK, D.; POWELL-COPE, G. Wheelchair-Related Falls in Veterans With Spinal Cord Injury Residing in the Community: A Prospective Cohort Study. American Journal of Physical Medicine \& Rehabilitation, v. 91, n. 8, p. 1166-1173. 2010. DOI: 10.1016/j.apmr.2010.05.008.

RICE, L. A..; PETERS, J.; SUNG, J.; BARTLO, W. D.; SOSNOFF, J. J. Perceptions of Fall Circumstances, Recovery Methods, and Community Participation in Manual Wheelchair Users. American Journal of Physical Medicine \& Rehabilitation, v. 98, n. 8, p. 649-656. 2019. DOI: 10.1097/PHM.0000000000001161.

RICE, L. A.; SUNG, J.; KEANE, K.; PETERSON, E.; SOSNOFF, J. J. A brief fall prevention intervention for manual wheelchair users with spinal cord injuries: A pilot study. The Journal of Spinal Cord Medicine, v. 43, n. 5, p. 607-615. 2019. DOI: 10.1080/10790268.2019.1643070. 
SILVEIRA, M. G. Descrição de acidentes com usuários de cadeira de rodas. 2019. Trabalho de Conclusão de Curso (Monografia)- Universidade Federal de São Paulo, Santos, 2019.

SINGH, H.; SCOVIL, C. Y.; BOSTICK, G.; KAISER, A.; CRAVEN, B. C.; JAGLAL, S. B.; MUSSELMAN, K. E. Perspectives of wheelchair users with spinal cord injury on fall circumstances and fall prevention: A mixed methods approach using photovoice. PLoS ONE, v. 15, n. 8, p. 1-22. 2020. DOI: 10.1371/journal.pone.0238116.

SINGH, H.; SCOVIL, C. Y.; YOSHIDA, K.; OOSMAN, S.; KAISER, A.; CRAVEN, C.; JAGLAL, S.; MUSSELMAN, K. E. Factors that influence the risk of falling after spinal cord injury: a qualitative photoelicitation study with individuals that use a wheelchair as their primary means of mobility. BMJ Open, v. 10, n. 2, p. 1-11. 2019. DOI: 10.1136/bmjopen-2019-034279. DOI: 10.1136/bmjopen-2019-034279.

SUNG, J.; SHEN, S.; PETERSON, E. W.; SOSNOFF, J. J.; BACKUS, D.; RICE, L. A; SUNG, J. H. Fear of Falling, Community Participation, and Quality of Life Among Community-Dwelling People Who Use Wheelchairs Full Time. Archives of Physical Medicine and Rehabilitation, v. 102, n. 6, p. 1140-1146. 2020. DOI: 10.1016/j.apmr.2020.06.025.

SUNG, J.; TRACE, Y.; PETERSON, E. W.; SOSNOFF, J. J.; RICE, L. A. Falls among full-time wheelchair users with spinal cord injury and multiple sclerosis: a comparison of characteristics of fallers and circumstances of falls. Disability And Rehabilitation, v. 41, n. 4, p. 289-395. 2017. DOI: 10.1080/09638288.2017.1393111.

TENNSTEDT, S.; HOWLAND, J.; LACHMAN, M.; PETERSON, E.; KASTEN, L.; JETTE, A. A Randomized, Controlled Trialof a Group Intervention To Reduce Fear of Falling and Associated Activity Restriction in Older Adults. Journal of Gerontology. Psychological Sciences, v. 53, n. 6, p. 384-392. 1998. DOI:10.1093/geronb/53b.6.p384.

THOMAS, L.; BORISOFF, J.; SPARREY, C. J. Manual wheelchair downhill stability: an analysis of factors affecting tip probability. Journal of NeuroEngineering and Rehabilitation, v. 15, n. 1, p. 95. 2018. DOI: 10.1186/s12984-018-0450-3.

UMMAT, S.; KIRBY, L. Nonfatal wheelchair- related accidents reported to the national electronic injury surveillance system. American Journal of Physical Medicine \& Rehabilitation, v.73, n.3, p. 163-167. 1994. DOI: 10.1097/00002060-199406000-00004.

XIANG, H.; CHANY, A-M.; SMITH, G. A. Wheelchair related injuries treated in US emergency departments. Injury Prevention, v. 12, n. 1, p. 8-11. 2006. DOI: 10.1136/ip.2005.010033. 